Carnets de géographes

GÉOGRAPHES.

\title{
Espaces résiduels et espaces collaboratifs : le développement du secteur socioculturel berlinois au sein des interstices de la ville (1990-2013)
}

\section{Elisa Goudin-Steinmann}

\section{(2) OpenEdition Journals}

Édition électronique

URL : http://journals.openedition.org/cdg/536

DOI : $10.4000 /$ cdg. 536

ISSN : 2107-7266

Éditeur

UMR 245 - CESSMA

Référence électronique

Elisa Goudin-Steinmann, «Espaces résiduels et espaces collaboratifs : le développement du secteur socioculturel berlinois au sein des interstices de la ville (1990-2013) », Carnets de géographes [En ligne] 7 | 2014, mis en ligne le 27 février 2019, consulté le 19 avril 2019. URL : http:// journals.openedition.org/cdg/536 ; DOI : 10.4000/cdg.536

\section{(c) (†) $\odot$}

La revue Carnets de géographes est mise à disposition selon les termes de la Licence Creative Commons Attribution - Pas d'Utilisation Commerciale - Pas de Modification 4.0 International. 


\title{
Espaces résiduels et espaces collaboratifs : le développement du secteur socioculturel berlinois au sein des interstices de la ville (1990-2013)
}

\author{
ELISA GOUDIN-STEINMANN \\ avec la collaboration de JULES-MATHIEU MEUNIER \\ Université Paris 3 - Sorbonne Nouvelle \\ Département d'Études germaniques \\ elisa.goudin-steinmann@univ-paris3.fr
}

\begin{abstract}
Résumé
Le tissu urbain s'est émietté au moment de la chute du Mur de Berlin, laissant apparaître de nombreux espaces "en attente ", qui ont été autant de laboratoires pour de nouvelles pratiques socioculturelles. Nous analysons, au moyen de quelques exemples (RAW-Tempel, Brotfabrik et Tacheles), le discours d'acteurs du monde socioculturel de l'ex Berlin-est sur leurs propres activités, la façon dont ils mettent en récit et en scène l'espace qu'ils ont investi en le détournant de son usage initial. L'une des conclusions est que pour ces personnes, l'espace n'est pas une simple topographie, il est en même temps le support d'une axiologie: on donne aux lieux une signification particulière par le biais de pratiques culturelles intrinsèquement liées à cet espace. Il y a une forme d'appropriation symbolique du lieu qui passe par la volonté d'exploiter sa singularité, de développer une correspondance entre ces terrains urbains interstitiels et des pratiques culturelles hors-normes.
\end{abstract}

\begin{abstract}
At the time of the fall of the Berlin Wall, numerous spaces appeared in Berlin and these spaces were used as laboratories for new sociocultural practices. We are analyzing with some examples (RAW-Tempel, Brotfabrik and Tacheles) how the actors of the sociocultural scene of the ex East Berlin describe their own activities, the narratives they use to talk about the space they invest far away from the initial use. One of the conclusions is that for them, the space is not only a topography, it also has a sense, a particular meaning intrinsically connected to these space. This is a symbolic appropriation of the in-between, witch tends to develop a correspondence between the interstitial urban grounds and new sociocultural practices.
\end{abstract}




\section{Introduction}

Les quarante années de division ont laissé dans la ville de Berlin de nombreuses places vacantes à la fois dans l'espace qui était occupé entre 1961 et 1989 par le mur, et aussi dans les nombreuses friches industrielles issues des anciennes entreprises de la RDA dont la production a été arrêtée, parfois brutalement, après la chute du régime du SED ${ }^{1}$, ou encore dans les anciennes maisons de la culture de RDA. Ces espaces physiques - ces interstices au sein de la ville - ont souvent été réutilisés, détournés de leur usage initial, pour devenir des lieux dédiés à l'animation socioculturelle et artistique. Le tissu urbain s'est émietté au moment de la chute du Mur de Berlin, et a laissé apparaître de nombreux espaces " en attente ", qui ont été autant de laboratoires pour de nouvelles pratiques culturelles, et notamment socioculturelles, où l'intermédiaire se conjugue avec le pérenne.

C'est en ce sens que nous définirons la notion d'entre-deux : des espaces qui ont perdu leur fonction sociale initiale et sont donc devenus des enjeux de lutte pour leur appropriation matérielle ou symbolique, pour reprendre la terminologie de Bourdieu. Par ailleurs, ces espaces en attente caractéristiques de Berlin-est après 1990 ont permis des cristallisations de la vie sociale, si l'on emprunte cette fois une terminologie durkheimienne, avec un nouveau lien social à construire par le biais de ces centres socioculturels ouverts à tous. La notion centrale est ici celle de détournement, ces espaces sont très souvent devenus des centres socioculturels en étant détournés de leur usage premier. Cela rappelle la définition que Pascal Nicolas-Le Start donne aux interstices au sein d'une ville comme des « espaces de questionnement » qui constituent la « réserve de disponibilité » d'une ville (Nicolas-Le Start, 2009). Le Berlin de l'après 1990, et c'est encore vrai en partie de nos jours, est une ville qui correspond parfaitement à cette définition : son développement un peu désordonné a laissé un grand nombre d'hypothèses non encore investies, favorisant le détournement au profit d'un projet socioculturel.

Notons que le terme de Soziokultur est présent en RFA (République fédérale d'Allemagne, I'Allemagne de l'Ouest) depuis les années 1970, mais n'était pas utilisé en RDA (République démocratique allemande, l'Allemagne de l'Est). C'est seulement après la chute du mur que le concept, ainsi que toutes les réflexions en matière de pédagogie culturelle qui l'accompagnaient, ont commencé à essaimer à l'est. Un grand nombre de centres culturels est-allemands ont pu survivre après 1990 grâce aux financements accordés par les instances centrales de l'Allemagne fédérale, sous l'appellation de centres "socioculturels ». En 1990, beaucoup ont constaté qu'ils menaient à bien des projets socioculturels depuis de nombreuses années mais qu'ils ne les avaient jamais nommés ainsi (Wagner, 2001). Ils se sont ajoutés aux nouveaux centres socioculturels issus le plus souvent de la scène alternative.

II nous a donc semblé intéressant d'étudier comment s'est organisée la réappropriation dans un objectif socioculturel des anciens cadres de vie typiques de la RDA qui avaient perdu brusquement leur fonction, en partant de l'analyse du discours d'acteurs du secteur socioculturel qui ont investi d'anciennes friches industrielles. Dans quelle mesure le secteur socioculturel berlinois, en reconvertissant ces espaces résiduels, a-t-il permis une nouvelle

1 Le SED, sozialistische Einheitspartei, issu de la fusion forcée entre les communistes et les sociauxdémocrates, était le parti au pouvoir en RDA, République démocratique allemande, entre 1949 et la chute du mur de Berlin. 
fabrique de la ville ? En quoi a-t-il permis de retisser des sociabilités, un vivre-ensemble qui avait été mis à mal par la fermeture brutale de nombreux lieux de création ? La volonté de faire exister un collectif va très souvent de pair avec le projet de monter un centre socioculturel, qui est aussi un lieu de vie en commun. Mais de quel "commun " parle-t-on alors ? II s'agira donc d'analyser les résultats de ces détournements d'espaces interstitiels laissés vacants par la chute du Mur, notamment les effets en termes de dynamiques urbaines, et aussi parallèlement d'analyser les logiques de justification des acteurs culturels telles qu'elles s'expriment dans leur discours. En effet, réinvestir des lieux abandonnés pour y installer un établissement culturel crée par définition des externalités positives, en attirant des personnes intéressées par cette offre culturelle, mais il y a aussi une autre dimension inhérente à cette démarche: ces espaces nouveaux pourraient être un vecteur d'embourgeoisement, selon une dynamique de gentrification, ce qui est perçu par de nombreuses personnalités du secteur socioculturel comme un risque de perte d'authenticité (Grésillon, 2002). Comment dès lors les acteurs du secteur socioculturel de Berlin-est ont-ils composé avec ces contradictions, comment ont-ils justifié leur démarche? En s'institutionnalisant, ces espaces ont profité d'un entre-deux à la fois spatial (détournement de friches, entre deux usages) et temporel (changement de régime politique, entre deux systèmes sociaux). Comment cet entre-deux a-t-il été propice au développement de pratiques nouvelles et quelles conséquences ces dernières ont-elles eu sur les dynamiques urbaines?

Afin de tenter d'apporter des réponses à ces questions, nous proposons de nous appuyer sur des sources de plusieurs natures: compte-rendus de débats à l'Assemblée nationale allemande, le Bundestag, sur les questions relatives au secteur socioculturel, archives d'associations socioculturelles berlinoises, en particulier I'Union nationale des centres socioculturels (Bundesvereinigung soziokultureller Zentren) qui regroupe huit centres pour la seule ville de Berlin, et entretiens avec deux acteurs du secteur socioculturel de cette ville ${ }^{2}$. Pour des raisons de clarté, nous avons choisi de n'étudier que des établissements culturels situés dans l'ex Berlin-est, même si des établissements comparables existent aussi dans l'ex Berlin-ouest.

Nous nous appuierons sur trois exemples concrets qui ont des histoires et des caractéristiques sensiblement différentes : le premier est très grand, il s'agit du RAW-Tempel, un centre socioculturel installé juste après la chute du mur sur une ancienne friche industrielle, dans le quartier de Friedrichshain, un quartier devenu à Berlin-est un quartier populaire dans les années 1980 mais qui est en train de devenir un quartier très prisé des étudiants et des artistes.

Le deuxième exemple est un centre socioculturel nettement plus petit mais installé dans le même quartier dans ce qui était avant la RDA une ancienne fabrique de pain et en RDA une maison de la culture de quartier.

Et le troisième exemple est internationalement connu: il s'agit du Tacheles, qui a disparu récemment mais a constitué pendant de nombreuses années un symbole de la créativité artistique de Berlin et du détournement d'espaces urbains. Les entretiens qui complètent l'analyse des documents produits par ces trois centres socioculturels ont été menés avec les responsables du RAW-Tempel, K. Schütt et de la Brotfabrik, J. Fügmann. Nous avons soumis

2 Entretien avec K. Schütt, Berlin, 23.01.2013, responsable du centre socioculturel RAW-Tempel et avec Jörg Fügmann, Berlin, 21.01. 2013, président du directoire de la Brotfabrik. 
à une même méthode d'analyse du discours les deux types de source, orales et écrites, afin de comprendre quels étaient les stratégies narratives mises en place pour parler de l'espace de l'entre-deux qu'ils occupent, détournent, imaginent, etc.

\section{Les spécificités du secteur socioculturel berlinois}

Comprendre l'acception allemande du terme socioculturel Soziokultur et ses origines intellectuelles est nécessaire pour comprendre comment ce courant d'idées ouest-allemand a pu être repris et décliné après 1990 afin d'influencer le redéploiement de certaines pratiques culturelles mises en œuvre en RDA. Des précisions permettent aussi d'évaluer pourquoi les espaces résiduels laissés vacants par la chute du Mur étaient particulièrement intéressants pour le développement du secteur socioculturel dans le Berlin réunifié.

Les origines de ce mouvement remontent aux années 1970, lorsque Hermann Glaser et Karl Heinz Stahl ont commencé à réfléchir en Allemagne à ce que serait une nouvelle culture (Glaser et Stahl, 1974) qui aurait une signification sociopolitique et servirait à démocratiser la société, et ceci sous l'influence, entre autres, de Herbert Marcuse et de ses analyses sur le caractère affirmatif de la culture à "l'époque bourgeoise". A contrario, le projet de la Soziokultur est de faire de la culture un outil pour renverser la tendance inégalitaire de la société: il s'agit de recréer de l'égalité des chances grâce au vecteur culturel, en encourageant toutes les formes de participation de larges franges de la société et en tournant le dos à une culture trop élitiste dont il est question de saboter l'hégémonie.

Le projet qui sous-tend le mouvement socioculturel en Allemagne n'est donc pas uniquement de revaloriser certaines formes de culture populaire face à une " haute culture ", il s'agit de se servir de la culture pour exercer une influence sur l'ordre social. De ce point de vue, la Soziokultur n'est évidemment pas une invention des années 1970 . Horst Groschopp par exemple fait remonter les origines de ce mouvement aux années 1890, où le discours progressiste de certains " dissidents " en marge de la société dominante a posé les bases d'une réflexion sur un concept élargi de la culture et sur une démocratisation des pratiques culturelles en Allemagne (Groschopp, 1997). Le caractère participatif de la Soziokultur est fondamental. II apporte une dimension politique nouvelle dans le contexte allemand, qui était plutôt caractérisé - à l'exception de la RDA et bien sûr du troisième Reich - par une tradition d'étanchéité, d'éloignement entre le politique et le culturel : "Ce projet de Soziokultur participative rompt par conséquent avec le modèle de pensée que portait la culture bourgeoise, avec "I'homme de culture apolitique» (Knoblich, $2001: 8$ ).

Les premiers théoriciens de la Soziokultur sont des partisans d'une plus grande politisation du débat culturel, ils veulent rompre avec l'hégémonie de la création culturelle comme domaine réservé, placé au-dessus de la société et donc coupé de cette dernière. Les racines de ce changement se situent dans les mouvements sociaux apparus dans les années 1970 : écologie, mouvements féministes, pacifistes, etc., dont la Soziokultur reprend le potentiel critique (Stüdemann, 1993 : pp. 220-235). L'objectif est de transformer la société en profondeur, en favorisant la rencontre entre les générations, entre les personnes issues ou non de l'immigration, entre les différentes couches sociales, etc., par le biais du travail culturel. L'absence de commercialisation est également une constante : il s'agit de définir une alternative à la culture dominante orientée vers la recherche du profit. Enfin, 
l'autogestion, la démocratie par le bas, la transparence, sont des valeurs importantes. On le voit, le préfixe "Sozio " n'est pas synonyme de "social ", il y a une réflexion beaucoup plus large sur le sens des pratiques culturelles pour la société allemande.

On comprend, dès lors, que de nombreuses maisons de la culture est-allemandes aient cherché à se reconvertir après 1990 en centres socioculturels. Les programmes de soutien à l'infrastructure culturelle est-allemande mis en place par la Fédération au moment de l'unification, notamment en 1994, comprenaient différents volets et l'un de ces volets était justement l'activité socioculturelle. Or, beaucoup de Kulturhäuser, de maisons de la culture est-allemandes, n'entraient dans aucune autre catégorie et ont donc logiquement tenté de trouver des financements en tant que centres socioculturels.

Au moment de l'unification, la tradition socioculturelle ouest-allemande a donc été confrontée à l'héritage de quarante années de politique culturelle socialiste en RDA. Assigner à la création artistique une mission sociale était en effet l'un des piliers de la politique culturelle de RDA, qui visait à réconcilier la tradition allemande de l'État culturel (Kulturstaat) avec une autre tradition allemande, celle du mouvement ouvrier (Arbeiterbewegung). De cette tentative de synthèse est né le concept de Breitenkultur que l'on pourrait traduire par "culture populaire " et qui fait référence à une culture qui s'adresse à tous et est également produite par le plus grand nombre. Ce terme s'oppose explicitement à la " haute culture " (Hochkultur) perçue comme élitiste. Le SED entendait utiliser les maisons de la culture pour développer un art authentiquement populaire, produit par les ouvriers et les paysans eux-mêmes, accompagnés par des professionnels. La différence entre art amateur et art professionnel devait à terme être abolie car perçue comme caractéristique du mode d'organisation d'une société capitaliste et bourgeoise. Le mouvement dit des "ouvriers écrivains", (schreibende Arbeiter), en est l'exemple le plus connu ${ }^{3}$.

\section{Spielräume (espaces de jeu) : Berlin et ses centres socioculturels}

Les deux héritages culturels réunis par la chute du mur avaient donc en commun de chercher à développer l'implication des citoyens. L'offre de culture devait être destinée au plus grand nombre et l'on attendait en retour une forme d'engagement de la part des acteurs de la vie socioculturelle : il s'agissait, en les impliquant au maximum, qu'ils ne soient plus seulement consommateurs de culture mais bien producteurs de biens culturels. Après 1990, l'enjeu est donc de reconvertir les anciennes maisons de la culture est-allemandes en réinvestissant ces espaces, ou en investissant de nouveaux espaces (friches industrielles par exemple) pour des pratiques culturelles alternatives. L'objectif est aussi social : on cherche à encourager la responsabilité individuelle et par le même mouvement, la responsabilité de chacun face à la communauté. La réunion des deux traditions culturelles, la Soziokultur ouest-allemande et l'héritage des maisons de la culture de RDA s'est effectuée sur la base

3 Ce mouvement des ouvriers écrivains est devenu emblématique de la politique culturelle de la RDA, il a donné lieu à de nombreuses expérimentations dans les usines (VeB), on a fait écrire des textes de différentes natures à des ouvriers, nouvelles, poèmes, pièces de théâtre, etc., le plus souvent sous la direction d'un écrivain " professionnel " mais toujours avec l'objectif affiché de gommer le hiatus entre écrivains professionnels et écrivains ouvriers. 
d'un dénominateur commun : I'aspect contre-sociétal. On voulait aller à rebours des valeurs d'individualisme et encourager les formes de création collectives et de responsabilité de chacun face au collectif. C'est en ce sens qu'il faut entendre la notion de pratiques culturelles " alternatives" qui fait le lien entre les projets issus de l'ex RDA et ceux issus du secteur socioculture ouest-allemand.

De tels objectifs impliquent un mouvement de transformation sociale des interstices, dans un contexte général de transformations urbaines intenses. Par exemple, le centre socioculturel Pfefferwerk Stadtkultur possède de nombreuses antennes dans Berlin, mais son siège est situé dans une ancienne brasserie classée monument historique du quartier de Prenzlauer Berg dans l'ex Berlin -Est, au nord de Berlin. Ce quartier a connu d'importantes modifications dans les années qui ont suivi l'unification, la très grande majorité des bâtiments sont aujourd'hui rénovés et accueillent de plus en plus de familles dont les parents sont diplômés, d'étudiants, et de célibataires travaillant souvent à domicile, et de moins en moins de familles ouvrières ou de personnes âgées. Ce centre socioculturel définit ainsi les objectifs qu'il s'est fixés:

"Nous fondons, conduisons, encourageons et mettons en réseau des projets et des institutions diverses de l'infrastructure sociale dans la région de Berlin. Notre offre et nos services sont guidés par une image de l'homme déterminée par les principes d'humanité, de liberté, d'égalité des chances et de solidarité. Ce faisant, nous nous attachons particulièrement à la responsabilité individuelle, à l'activité des individus et à la responsabilité de chacun face à la communauté (Pfefferwerk Stadtkultur gGmbH, 2013) ».

Il apparaît clairement ici que l'objectif de ce centre culturel est aussi de retisser des sociabilités, d'encourager la réfection des solidarités entre citoyens, en misant sur l'implication de ces derniers. L'espace du centre socioculturel doit être un espace collaboratif, que les citoyens sont à même de s'approprier (Baudry, 2012).

C'est la raison pour laquelle les lieux laissés à l'abandon après la chute du Mur ont été très souvent une cible privilégiée pour les acteurs du monde socioculturel en quête d'espaces provisoires. Le grand centre socioculturel RAW-Tempel s'est par exemple installé sur une friche industrielle et est devenu en quelques années un temple de la culture alternative à Berlin. Le terme de " culture alternative ", Alternativkultur, est d'ailleurs présent dans le mot d'introduction rédigé pour la brochure de présentation de RAW-Tempel (RAW-Tempel, 2010) par Kristine Schütt ${ }^{4}$ qui dirige ce centre avec trois autres artistes, et elle a également utilisé plusieurs fois ce terme dans l'entretien qu'elle nous a accordé.

Le terme de culture alternative a un sens qui peut varier selon les usages. Dans le contexte berlinois de l'après-unification, il désigne le plus souvent de façon générale les expérimentations artistiques ou culturelles en marge de la culture officiellement reconnue, financée, à l'écart des médias de masse, voire même en marge de la société. C'est en ce sens que le terme a été employé par K. Schütt. Une culture alternative ne peut pas, selon elle, se développer sans espaces de jeu, "Spielräume ", et le RAW-Tempel en est un. L'endroit sur

4 Elle est musicienne et danseuse et connue sous le nom d'artiste Mikado, elle fait partie de la scène underground berlinoise et est impliquée dans le projet de RAW-Tempel depuis 1999. Elle a choisi de s'installer dans ce lieu en raison des possibilités presque infinies qu'il offrait, car de nombreuses salles avec des acoustiques différentes permettaient de mener à bien des expérimentations variées. 
lequel ce centre socioculturel s'est installé est important : les grandes salles désertes laissées à l'abandon se prêtaient à toutes formes d'expérimentation, notamment pour la danse et la musique, car l'acoustique était selon elle très intéressante. De plus, cet espace était à géométrie variable, on pouvait transformer à l'envi l'enchevêtrement de salles en bistrots, scènes de théâtre, salles de projection de cinéma, etc. Le caractère provisoire de cet espace est donc pour $\mathrm{K}$. Schütt intrinsèquement lié à la démarche alternative, même si le provisoire a finalement tendance à se consolider, puisque le centre socioculturel existe maintenant depuis une vingtaine d'années. Cela s'explique par le fait qu'en dépit de cette pérennité, l'existence du centre est chaque année remise en cause et il est très difficile pour les personnes qui y travaillent de se projeter dans l'avenir. Ce n'est pas un hasard si notre interlocutrice a utilisé de façon récurrente le terme de "Spielräume ", espaces de jeu. On peut en effet y entendre le double sens d'espaces de jeu, donc de salles pour jouer, expérimenter, et de jeu sur l'espace, sur les différentes possibilités ouvertes par ces spatialités hors normes. Plusieurs études montrent comment certains lieux ont un pouvoir particulier de retentir, de "ricocher en I'homme " (Sansot, 2004). Le jeu sur cet espace est donc bien un jeu sur l'entre-deux : on utilise le fait que le lieu ne soit pas réductible à une catégorie bien spécifique, les salles du RAW-Tempel n'ont pas une fonction unique et définitive. On se refuse à normer l'espace afin de rester, au moins sur le plan symbolique, dans une forme d'entre-deux qui s'apparente ici à une indécision sur la nature de l'espace.

Le nom de Tempel (temple) a été choisi car l'un des bâtiments ressemblait à une chapelle en raison de sa forme arrondie. Les initiales RAW signifient quant à elles "Reinkommen, Ankommen, Wiederkommen ", c'est-à-dire " entrer, arriver, revenir ». Cette dénomination met bien en évidence un pilier sur lequel est fondé le RAW-Tempel selon K. Schütt : la priorité absolue est l'expérimentation, il faut que les projets restent provisoires, que l'on cherche sans cesse à faire du nouveau. Lors de notre entretien, il était clair qu'il existait pour elle une contradiction indépassable entre le fait de s'établir en investissant de façon définitive un espace, et le fait de rester créatifs, de conserver une ouverture suffisante. Elle utilise le terme " etabliert " pour désigner cette tendance à s'établir. Selon elle, l'entre-deux ne peut pas s'institutionnaliser sans que cela n'aille de pair avec une perte de créativité. Et une telle recherche de l'ouverture, de l'expérimentation, de la nouveauté, ne peut être menée à bien dans un espace trop restreint, ou aux contours trop fixes. L'intérêt de cette friche industrielle est qu'elle était malléable presque à l'infini. Elle représentait une brèche dans le tissu urbain qui n'était pas prédéterminée, en sorte que l'on pouvait l'investir de plusieurs façons. L'unicité, l'irréductibilité à la normalisation, sont des caractéristiques importantes de ces espaces. Tous nos interlocuteurs ont souligné la singularité du lieu dans lequel ils travaillaient.

On constate donc que la dimension du jeu est une dimension essentielle de l'utilisation d'un espace considéré comme un entre-deux: dans les brèches que contient le tissu urbain berlinois, davantage encore que d'autres tissus urbains, il a été possible d'installer des Spielraüme, des espaces de jeux pour encourager des formes de participation des citoyens et de créativité artistique en marge de la culture institutionnalisée. 


\section{Mise en récit et mise en scène du lieu interstitiel : maintenir l'entre-deux ?}

On constate également que le discours sur le type d'espaces investis est intéressant : il y a une mise en récit de l'espace qui prend la forme d'une conquête de l'espace. Le discours contient souvent une forme de positionnement offensif face à l'espace qui va être investi pour des activités socioculturelles, avec l'idée de détourner, de s'approprier cet espace, d'en proposer une lecture inédite ou même de le rendre lisible alors qu'il était auparavant relativement illisible, ou perçu comme tel. Le lieu est conçu comme un espace à défricher avec un lien social à construire, et c'est la raison pour laquelle la dimension de l'entre-deux est importante : dans la mesure où il n'y a pas de lecture arrêtée, unique, définitive, du lieu car il se situe dans un entre-deux, on peut l'investir. L'entre-deux se construit alors en marge du reste de la ville, et doit donc par nature tenter de résister à toute forme d'institutionnalisation qui remettrait nécessairement en cause cet entre-deux.

Dans le même temps, on peut remarquer que le discours des responsables de centres socioculturels de Berlin-est est régi par une volonté de justification. K. Schütt par exemple est consciente des effets secondaires inhérents à la démarche de réappropriation des friches industrielles : le quartier de Friedrichshain où est situé le RAW-Tempel est un quartier de I'ancien Berlin-est directement à l'est du centre historique, l'axe principal ouest-est est dominé par la Karl-Marx Allee réalisée au milieu des années 1950 selon les principes du réalisme socialiste. Dans les années 1980, l'introduction d'immeubles de béton (Plattenbau) a fait de ce quartier un quartier plutôt populaire, mais qui aujourd'hui est prisé par la population étudiante et de nombreux artistes souvent venus de Mitte ou de Prenzlauer Berg. Des processus de gentrification sont perceptibles, avec le développement du tourisme, la hausse des valeurs immobilières, etc.

Depuis la refonte administrative de 2001, Friedrichshain forme avec Kreuzberg l'un des 12 districts (Bezirke) de Berlin (Grésillon, 2002 et Boichot, 2011). Or, K. Schütt sait que les ambitions du secteur socioculturel dans le domaine social peuvent être mises à mal par les effets secondaires du détournement d'espaces vacants, car cela change progressivement le visage de ces territoires péri-centraux et risque donc d'en chasser certaines catégories défavorisées. La question est donc de savoir comment justifier le projet du RAW-Tempel pour qu'il soit conforme aux idéaux de départ, si l'on assume un point de vue normatif qui consiste à penser, comme $\mathrm{K}$. Schütt, que la création du centre socioculturel ne sera un objectif vertueux que s'il ne s'effectue pas au détriment de la mixité sociale. C'est la raison pour laquelle on retrouve des logiques de justification dans le récit de l'utilisation de l'espace par notre interlocutrice : nous créons un lieu alternatif qui va drainer une utopie, apporter du lien social, permettre de tisser des sociabilités nouvelles, et non pas uniquement faire monter le prix des loyers dans le quartier concerné. L'avantage à laisser ces lieux de création dans un entre-deux et à refuser autant que possible de les institutionnaliser est donc double, car fondé sur des arguments de nature politique (le risque de mettre à mal la mixité sociale) et de nature artistique (la nécessité d'utiliser des espaces hors-normes pour encourager la créativité).

Une correspondance symbolique s'installe alors entre le type de lieu utilisé et les activités socioculturelles. Ainsi, l'objectif du RAW-Tempel est de faire émerger une culture qui soit une « contre-proposition à une conception de la culture purement consumériste, orientée 
uniquement vers le profit et le divertissement (RAW-Tempel, 2010) ». L'opposition avec ce qui est produit dans le cadre de la " haute " culture, ou de la culture institutionnalisée, est très prononcée dans les propos de Kristine Schütt. Cela se lit aussi dans le texte de présentation de cette association sur leur page internet :

"Le RAW-Tempel se conçoit comme un lieu de travail et d'expérimentation pour des projets novateurs visant le développement participatif, et pour des modèles sociaux et économiques alternatifs organisant une communauté autonome. Le RAW-Tempel se conçoit comme une organisation ouverte, d'intérêt général, réunissant des citoyens et des personnes créatives, qui partagent une vision : bâtir un espace libre pour une utilisation socioculturelle au cœur d'une métropole européenne, dans lequel la réalisation de soi passe par une action solidaire pour le collectif (RAW-Tempel, 2013) ».

La notion de développement participatif sert donc de base à la réflexion autour des possibilités de vie en commun alternatives. II s'agit de fonder un " espace libre " qui abrite une "communauté autonome ": le RAW-Tempel se conçoit donc en quelque sorte comme un "dehors en dedans", un "à-côté " au cœur de la ville. Le projet de ce centre socioculturel est très lié aux spécificités du lieu et s'accompagne d'un discours précis élaboré autour de cette notion d'un à-côté interne à la ville. L'entretien que nous avons mené avec K. Schütt fait apparaître une discontinuité marquée, la volonté de se situer dans un espace libre, autonome, en rupture avec la culture institutionnalisée. II s'agit de mettre à profit cet « en dehors", d'utiliser un repli de la ville de Berlin pour y développer un vivre-en-commun alternatif. L'objectif ne se limite pas à fabriquer de l'art sous différentes formes, mais bien à favoriser l'émergence d'un "vivre ensemble » différent. Le RAW-Tempel est d'après ses statuts un centre socioculturel, mais il est décrit ici comme une communauté autonome rassemblant des citoyens qui cherchent des modèles sociaux et économiques alternatifs.

C'est bien un lieu de vie autant qu'un lieu de création. On peut souligner la polysémie du terme de "repli » au sein de la ville : un lieu un peu à la marge à la fois d'un point de vue géographique, puisque le RAW-Tempel est situé dans le quartier de Friedrichshain ${ }^{5}$, et d'un point de vue institutionnel. En somme, l'entre-deux est défini ici comme une tension entre les deux pôles théoriques que seraient la culture strictement institutionnalisée, officielle, soutenue par les médias de masse d'une part, et le refus total de s'inscrire dans la ville et dans ses traditions culturelles en se plaçant délibérément en dehors de tout cela d'autre part. C'est la raison pour laquelle nous proposons l'expression de repli, ou encore l'idée d'un « dehors en dedans » pour déterminer les caractéristiques de ces espaces de l'entre-deux.

L'utilisation même du lieu exprime ces propositions: il s'agit souvent d'un lieu très ouvert, où de nombreux artistes passent une grande partie de leur journée, où des personnes viennent prendre un verre pour un prix extrêmement bas, car le bar ne réalise presque pas de profit, etc. Les pratiques culturelles sont également accessibles au plus grand nombre : à titre d'exemple le cours de céramique proposé au RAW-Tempel ne coûte en 2013 que 2,5 euros de l'heure. En suivant la même logique, la Brotfabrik, un autre centre socioculturel berlinois, propose aussi de nombreux ateliers gratuits, en particulier pour les jeunes, et l'accès gratuit à toutes les manifestations pour les personnes vivant des minima sociaux. II s'agit par là de viser le plus large public possible, et donc de reprendre l'un des fils rouges du concept de

5 Le RAW-Tempel a été fondé dans un ancien dépôt ferroviaire de la Revaler Strasse, sur un espace de 6000 $\mathrm{m} 2$ au total, qui appartiennent à la DB, l'équivalent allemand de la SNCF. 
Soziokultur tel qu'il s'est développé dans les années 1970. L'innovation spatiale va donc de pair avec une forme d'innovation sociale. De plus, il s'agit de se réaliser par le collectif, de former une communauté de vie, le travail des autres artistes étant source d'inspiration pour soi. On comprend dès lors pourquoi le champ lexical de l'ouverture aux autres, celui de la liberté et de la vision sont si présents dans les documents de présentation de ces centres.

La mise en scène et la mise en discours du lieu participent à sa visibilité et interrogent les possibilités de sa reconnaissance par les pouvoirs publics. En effet, le RAW-Tempel existe depuis 1999 et après deux tentatives avortées pour y installer un squat d'artistes, l'endroit est maintenant utilisé de façon légale, dans le cadre d'un contrat à court terme avec la ville de Berlin, qui doit sans cesse être renouvelé. La construction du lieu fortement transgressive au départ a donc laissé place à une construction de l'espace moins transgressive aujourd'hui. L'avantage de cette reconnaissance est que les occupants ne doivent s'acquitter que des charges, Betriebskosten, et ne payent pas de loyer. Mais le revers de la médaille est qu'ils doivent résoudre eux-mêmes tous les problèmes et, surtout, que l'avenir est très incertain. Kristine Schütt considère que l'incertitude permanente et la recherche d'argent est " épuisante », et est très critique envers les autorités de Berlin.

Le même rapport compliqué du secteur socioculturel avec les pouvoirs publics se retrouve dans un autre exemple de centre socioculturel berlinois, celui de la Brotfabrik. Ce centre se nomme ainsi car il est installé dans une ancienne fabrique de pain, ce qui constitue un autre exemple de détournement de l'usage d'un lieu'. Jörg Fügmann, qui fait partie de la direction, nous a accordé un entretien, dans lequel il insistait sur la contradiction entre le fait de rester dans une forme d'indépendance et de recherche de l'expérimentation d'une part et la nécessité de tisser des liens avec le monde politique pour obtenir des financements d'autre part. Il pense que des institutions socioculturelles comme la Brotfabrik ont permis de sauver la scène culturelle off de la RDA lors du passage vers l'économie sociale de marché.

Selon lui, il n'existe plus aujourd'hui à Berlin d'endroits libres - «Freiräume " - pour permettre à de telles expériences culturelles d'exister : tous les bâtiments de la ville ont désormais un propriétaire et il devient presque impossible d'envisager des occupations de lieux pour les transformer en ateliers de création culturelle. Il ajoute que même si cela existait et qu'un collectif d'artistes tentait de squatter un lieu, ils seraient aujourd'hui immédiatement expulsés, ce qui contraint à négocier avec les milieux politiques, à faire un travail pour se faire accepter sur la scène publique. Jörg Fügmann affirme ainsi consacrer une grande partie de son temps au travail de lobby politique. Interrogé sur la distance par rapport à l'État, il répond qu'elle lui semble impossible à maintenir, même s'il le regrette: " nous ne pouvons pas nous tenir à distance de l'État ». Il est nécessaire de composer avec les autorités de la ville, avec qui le dialogue n'est pas toujours facile. En somme, Jörg Fügmann regrette que les considérations non marchandes ne guident plus suffisamment l'intervention urbaine à Berlin.

6 Cette fabrique de pain est située dans le quartier de Weisensee, à coté de Prenzlauer Berg, donc dans I'ancien Berlin-est, et fait aujourd'hui partie du district de Pankow. Elle se situe sur la place Caligari, Caligariplatz, nommée ainsi car le film Das Cabinet des Dr Caligari de Robert Wiene y a été tourné en 1920. 
Les exemples de la Brotfabrik et du RAW-Tempel montrent que les espaces dédiés à I'animation socioculturelle ont souvent été des espaces détournés de leur usage initial et qui sont devenus aujourd'hui des espaces en tension avec l'espace public.

Ces remarques posent plus largement la question du degré de soutien manifesté par les pouvoirs publics à l'égard de la scène socioculturelle au niveau national. En Allemagne, le soutien à la culture est la prérogative quasi exclusive des Länder, mais on observe également - depuis la création en 1998 d'un ministère d'État aux affaires culturelles - une réflexion sur ces sujets menée au niveau de la Fédération. Depuis l'unification, deux "grandes questions " ont été adressées au gouvernement par des membres de l'Assemblée nationale allemande sur ce sujet, et ont donc suscité deux réponses du gouvernement sous forme de textes en 1990 (Deutscher Bundestag, 1990) et en 1999 (Deutscher Bundestag, 1999). La teneur de ces deux textes est très consensuelle, le gouvernement soulignant l'importance et la qualité du travail culturel effectué dans les centres socioculturels. Lorsque les députés, dans la question datée de 1999, évoquent le faible engagement de l'État et demandent au gouvernement s'il a l'intention de promouvoir davantage le secteur socioculturel, la réponse du gouvernement est contrastée : le fait que ces centres se financent en grande partie euxmêmes est défini comme un gage d'indépendance par rapport à l'État. Au total, l'autogestion permettrait "d'assurer l'indépendance politique, économique et organisationnelle vers l'extérieur - qui fait partie du credo des centres socioculturels - ainsi que l'autonomie souhaitée dans la définition des contenus en interne (1999: 24)». L'État présente donc son faible soutien financier comme une garantie d'indépendance! D'une façon générale, on constate que le secteur socioculturel est encore souvent assez peu reconnu par les pouvoirs publics, aussi bien au niveau national que dans le cas particulier de Berlin, même si le discours est souvent très favorable à ce secteur.

\section{Les enjeux de la pérennisation des interstices pour le développement de l'identité urbaine}

Même si la géographie urbaine de Berlin a fortement changé depuis 1990, certains centres socioculturels ont réussi à s'installer dans la période où le flou régnait sur les questions de propriété des nombreux espaces laissés au sein de la ville par la chute du régime du SED, et à rester actifs par la suite. Pour ces établissements comme pour les autres, l'identification avec la ville de Berlin est très forte et justifie leur pérennisation. L'exemple du Förderverband Kulturinitiative est intéressante à cet égard ${ }^{7}$ :

« Förderverband est une association d'utilité collective fondée en novembre 1989 à Berlin-est, pour permettre un travail culturel indépendant (...) il fait partie à part entière de la scène culturelle libre et représente un facteur décisif de stabilisation de son infrastructure au sens du maintien d'un paysage culturel décentralisé aussi diversifié que possible à Berlin (CD Förderverband, 2005) »

Au départ, Förderverband était une initiative regroupant en 1989 des artistes est-allemands, dont l'objectif était d'utiliser autant que possible les nouvelles marges de liberté. Certains

7 Förderverband Kulturinitiative Berlin est un autre membre de la Fédération des centres socioculturels qui soutient différents projets artistiques de la scène berlinoise. Il est situé lui aussi dans l'ex Berlin-est, dans la rue Torstrasse, quartier de Mitte. 
étaient anonymes, mais d'autres étaient déjà connus, tels Christa Wolf, Heiner Müller, Hermann Beyer, Christoph Hein. Tous avaient en commun une volonté de renouveler les paradigmes culturels, de profiter de l'occasion offerte par la chute du mur, comme le dit Catherine Cremer, membre fondateur de l'association :

"Le mois d'octobre nous avait ouvert l'esprit... nous n'avions qu'une vague idée de la force des rêves qui était en nous. Nous avions tout simplement un désir immense de faire quelque chose, et c'est de ce désir qu'est né Förderverband (CD Förderverband, 2005)».

La brochure du Förderverband met ainsi en exergue une phrase de Christoph Hein : «1989: c'était une période très intense, où l'on ne pouvait pratiquement pas dormir (CD Förderverband, 2005)».

Les conditions propres à Berlin en 1989/90, avec ses nombreux interstices au sein de la ville, ces espaces résiduels laissés vacants par la chute rapide de la RDA, ont donc considérablement influencé le développement et l'institutionnalisation de cette scène culturelle libre : " En même temps une scène culturelle off très diversifiée s'est épanouie - la ville était en plein essor et en pleine mutation, stimulante, ouverte (CD Förderverband, 2005 : p. 11) ". Le Berlin de la transition est donc présenté comme un terreau particulièrement propice au développement de nouvelles formes de travail culturel en marge de ce qui s'était fait auparavant.

Cela explique aussi probablement pourquoi la période de la fin de la RDA reste dans la mémoire des principaux acteurs du secteur socioculturel berlinois comme une période particulièrement heureuse. J. Fügmann nous a dit par exemple : "Les six derniers mois de la RDA ont été les plus heureux de notre vie ». Le pronom " notre " fait ici référence à cet élan collectif qui animait une grande partie des artistes et acteurs du travail culturel de RDA. A cette époque, les autorités de RDA avaient totalement abandonné tout système de contrôle ou de répression à l'égard de la scène culturelle, et en même temps la RFA n'avait pas encore son mot à dire dans la mesure où la RDA était encore officiellement en place. Les dynamiques juridiques qui allaient se mettre en place suite à l'unification n'étaient pas encore présentes. C'est ainsi que le projet de la Brotfabrik a pu voir le jour sans aucun obstacle administratif.

La restructuration aurait selon Jörg Fügmann nécessité plusieurs années dans le contexte de la RFA, alors qu'en 1989, il leur a suffi d'informer les autorités est-allemandes de la refonte de l'ancienne institution, à travers ce qu'il a nommé " eine freundliche Übernahme ", une OPA amicale. De la même façon, D. Roewer, qui coordonne le travail du Förderverband, met en avant la densité de rêves et d'énergies qui a caractérisé cette période intermédiaire à Berlin, et qu'on peut résumer par un concept récurrent dans ses propos, celui de Aufbruchstimmung. Le terme est difficile à traduire, il indique une atmosphère de créativité, de renouveau, porteuse d'espoir, d'élan, où l'occupation des lieux et leur détournement pour un usage culturel ne posait pas de problème administratif ni juridique.

Ce contexte explique aussi pourquoi l'engagement militant pour la Soziokultur est souvent associé à Berlin à la définition de projets de vie alternatifs, qui reviennent régulièrement dans le discours de ceux qui pratiquent ces espaces résiduels et deviennent peu à peu symboles voire synonymes de l'identité berlinoise. Ainsi, l'un des habitants du squat "Rauchhaus " occupé depuis plusieurs années par des artistes qui organisent des activités socioculturelles se décrit comme animé par une volonté de prolonger le mouvement hippie tel qu'il s'était développé en RDA (Rauchhaus, 2013). De la même façon, le projet du 
Tacheles était associé à une volonté de fonder un foyer qui soit à la fois un lieu de création artistique et un lieu de vie. Le Tacheles est le nom donné à un grand squat d'artistes venus du monde entier et associé à un symbole de la créativité artistique et du détournement d'espaces. Malheureusement les efforts pour sauver cette institution n'ont pas abouti, le Tacheles est maintenant fermé, ce qui en a également fait un symbole de la résistance vaine contre la pression des promoteurs immobiliers. Le Tacheles avait l'ambition de constituer un " contre-pôle à la culture dominante établie et institutionnalisée (Tacheles, 2012)». Pour devenir un tel contre-pôle à la culture dominante, la notion d'expérimentation a été mise en avant : le Tacheles se définissait lui-même comme étant " quasiment un laboratoire artistique, et ainsi aussi bien le lieu que le contenu de la création artistique (Tacheles, 2012)». Le rapport de métonymie est donc entre le contenu et le contenant : le Tacheles en tant que bâtiment est un objet artistique et aussi un lieu dans lequel on fabrique des objets artistiques : un espace de rupture, de discontinuité dans le paysage urbain, dans lequel peuvent s'épanouir des pratiques artistiques alternatives. Cette métonymie entre le contenu (les pratiques socioculturelles) et le contenant (les espaces résiduels, en rupture, le dehors en dedans) est décisive. Dans le discours des acteurs des différents centres socioculturels que nous avons pu interviewer, on retrouve de façon récurrente des références à l'importance du lieu : le développement de pratiques artistiques alternatives, et d'un nouveau vivre-ensemble, présupposait le choix d'un lieu non-conventionnel, d'un espace littéralement consacré (Grésillon, 2002 et 2004).

Le mot Tacheles vient du yiddish et signifie " s'expliquer, se dévoiler, parler franchement ". Au départ, un groupe de musiciens en RDA s'est donné ce nom programmatique, avec l'idée de développer des modes d'expression plus libres. Puis le projet a pris une grande ampleur, au point de devenir connu sur la scène artistique internationale :

"Au cours de ses années d'existence, la maison d'art Tacheles est devenue une œuvre d'art globale complexe. Cela se voit dans le fait que le Tacheles est devenu, bien au-delà des limites de Berlin, un symbole de la situation du Berlin réunifié et de l'élan vers un présent artistique nouveau, libéré en grande partie des considérations mercantiles (Tacheles, 2012) ».

Cette institution semble ainsi s'identifier avec la ville de Berlin, selon une métonymie entre le centre culturel et la ville : Berlin, tout comme le Tacheles, refuserait les considérations mercantiles, comme Berlin, le Tacheles se situerait dans un élan artistique. De nombreux documents insistent également sur le fait qu'un tel lieu ne pouvait voir le jour qu'à Berlin. En somme, le Tacheles, qui est membre de la Fédération nationale des centres socioculturels, incarne ce que la ville a à offrir en matière de liberté, d'ouverture, etc. Selon un mouvement de légitimation mutuelle, le Tacheles devient l'un des symboles de la ville à l'extérieur et la ville de Berlin elle-même inspire un certain état d'esprit auquel le centre culturel s'efforce de rester fidèle. Au-delà, le Tacheles participe donc au projet de communication municipale, tandis que la ville est essentielle à la publicité du centre.

Ainsi, les centres socioculturels berlinois se sont donc adaptés à la ville en pleine mutation, en s'installant dans les interstices qu'elle offrait et en profitant de ces opportunités spatiales pour définir un projet qui a pu être pérennisé. D. Roewer l'explique dans le CD du Förderverband au moyen d'une métaphore surprenante: "Nous sommes comme une amibe. Les amibes sont en quelque sorte le contraire des navires de guerre blindés, elles sont petites, ont des talents variés, sont flexibles et peuvent changer en permanence leur forme pour s'adapter aux conditions extérieures (2005: 19)». Cette pérennisation des centres socioculturels, malgré les contradictions qu'elle engendre en termes de dynamiques 
urbaines et de relations aux pouvoirs publics, est aujourd'hui indissociable de la construction de l'identité berlinoise.

\section{Conclusion}

Pour les centres socioculturels RAW-Tempel, Brotfabrik et Tacheles, l'espace n'est donc pas une simple topographie, il est en même temps le support d'une axiologie : l'espace fait sens, il prend une signification particulière par le biais de pratiques culturelles et de modes d'appropriation intrinsèquement liés à cet espace. Les trois exemples analysés ont tous cherché à exploiter la singularité de leur lieu, à développer une correspondance entre ces terrains urbains interstitiels et des pratiques culturelles hors-normes, innovantes, hétérotopiques. L'idée est d'utiliser un espace non-normé, ouvert, qui n'est pas figé ou prédéterminé, pour résister à l'homogénéisation des pratiques culturelles, en amorçant, en initiant des pratiques culturelles conçues pour rester flexibles, temporaires, réversibles.

Dans cette optique, ce n'est pas un hasard si le premier congrès de la Fédération nationale des centres socioculturels allemands postérieur à l'unification a été organisé à Berlin-est en 1992 sous le titre Utopien leben, "vivre les utopies ». L'utopie d'un modelage de la société par l'activité artistique est en effet une constante dans les réflexions sur le développement de la Soziokultur. II s'agit de faire exister un collectif, de réaliser des expérimentations culturelles mais aussi sociales, en encourageant toutes les formes de développement participatif. Le détournement d'espaces vacants doit encourager, favoriser, l'émergence de ces nouveaux liens sociaux et de ces nouvelles pratiques culturelles, les deux aspects étant indissociables. Or, le préalable est que l'espace utilisé soit un espace adapté, au sens où il serait pas un espace normé, mais bien un espace en attente, comme une hypothèse non encore investie.

L'analyse du discours des acteurs du monde socioculturel et de la façon dont ces centres mettent en récit leur projet montre que l'entre-deux possède pour eux deux dimensions intrinsèquement liées : il y a un entre-deux spatial, la volonté d'utiliser un espace ouvert, non réductible à une dénomination claire et définitive, en détournant un espace qui n'était pas conçu au départ pour des activités culturelles. Et il y a aussi un entre-deux temporel, historique, lié aux possibilités offertes par la transition entre deux régimes. Une étude commandée par le Sénat de Berlin et publiée en 2007 portait sur les espaces utilisés de façon provisoire à Berlin. Cette publication s'appuyait sur les résultats de deux projets de recherche, nommés Urban Catalyst et Raumpioniere (Urban pioneers, 2007), et était arrivée à la conclusion que l'utilisation temporaire d'espaces - leur analyse ne portait pas sur le secteur culturel - était en train de transformer Berlin. La chute du mur de Berlin a en effet engendré un émiettement du tissu urbain, laissant apparaître de nombreux espaces interstitiels au sein de la capitale. La reconnaissance de ces espaces de l'entre-deux est un marqueur important de l'identité de la ville de Berlin, au point que les squats d'artistes ont été utilisés comme argument de communication, dans une métonymie entre la ville et le projet de détournement d'espaces vacants.

Mais au-delà des caractéristiques propres au Berlin de l'après unification, cette ville nous permet de comprendre, plus généralement, comment une correspondance symbolique peut s'établir entre un espace de l'entre-deux et une pratique culturelle innovante, dans le 
discours des usagers de cet espace. Ce discours, nous l'avons souligné, contient aussi ses propres contradictions et apories. Les questions soulevées par la tendance à l'institutionnalisation des espaces de l'entre-deux sont par exemple en contradiction avec le projet initial d'utilisation temporaire, réversible. Au delà du cas berlinois, l'analyse de ce discours permet donc de comprendre comment, dans l'utilisation de ces espaces de l'entredeux, l'intermédiaire peut se conjuguer avec le pérenne. 


\section{Bibliographie}

BAUDRY P. (2012), La ville, une impression sociale, Belval, Circé, coll. L'Art de ne pas, 120 p.

Deutscher Bundestag, 11. Wahlperiode, Grosse Anfrage Soziokultur, réponse du gouvernement : Drucksache 11/6971 datant du 25. 04. 1990.

Deutscher Bundestag, 14. Wahlperiode, Grosse Anfrage Soziokultur, Drucksache 14/1575 datant du 07.09.1999, Drucksache 14/4020 du 20. 08. 2000.

BOICHOT C. (2011), Centralités artistiques et recomposition des espaces urbains : les enjeux d'une géographie de l'art à Paris et à Berlin, Mondes du tourisme, pp. 350-361.

BOURDIEU P. (1979), La distinction, Critique sociale du jugement, Paris, Editions de minuit, coll. Le sens commun, $672 \mathrm{p}$.

CD Förderverband Kulturinitiative e.V., 1989/90-2005, Tonusarcus, 2005.

GLASER H. et STAHL K-H. (1974), Die Wiedergewinnung des Ästhetischen. Perspektiven und Modelle einer neuen Soziokultur, München, Juventa Verlag, München.

GRESILLON B. (2002), Berlin métropole culturelle, Belin, Paris, 352 p.

GRESILLON B. (2004), « Histoire d'un « SquArt » berlinois: la Tacheles », in: Multitudes, N¹7.

GROSCHOPP H. (1997), Dissidenten. Freidenkerei und Kultur in Deutschland, Berlin, Dietz Verlag, $448 p$.

HILDEGART K. "Soziale Plastik heute ", magazine Oya anders denken, anders leben, entretien de l'auteure avec Shelley Sacks, 09/2011

HÜBNER E., Brochure de présentation, Förderverband Kulturinitiative Berlin

KNOBLICH T. , "Das Prinzip Soziokultur - Geschichte und Perspektiven", in : Aus Politik und Zeitgeschichte, B11/2001.

NICOLAS-LE START P. (2009), Expérimentations politiques, éditions Fulenn, 120 p. (notamment le premier chapitre intitulé « Multiplicité interstitielle »).

Pfefferwerk Stadtkultur, Leitbild, brochure de description du centre socioculturel,

http://www.pfefferwerk.de/index.php/pfefferwerk-stadtkultur-ggmbh/leitbild, consulté le 15 décembre 2013.

Rauchhaus, centre abritant des artistes du milieu socioculturel,

http://www.positiverage.com/BERLIN/berlin03.html, consulté le 14 septembre 2013 
RAW-Tempel (2010), 1998-2010, 12 Jahre RAW-Tempel e. V., Eine Chronik, 40 p.

RAW-Tempel, Page internet du centre socioculturel RAW-Tempel, www.raw-tempel.de, consultée le 17. 11. 2013.

SANSOT P. (2004), Poétique de la ville, Petite bibliothèque Payot, Paris, 626 p.

STÜDEMANN J. (1993), „Soziokulturelle Zentren im Umfeld der Neuen Sozialen Bewegungen. Die bundesdeutsche Situation", in: SCHWENKE O. (ed.), Kulturelle Modernisierung in Europa. Regionale Identitäten und soziokulturelle Konzepte, Hagen, Klartext Verlag, Edition Umbruch, pp. 220-235.

TACHELES, Site internet du Kunsthaus Tacheles, www.Tacheles.de, consulté le 7 août 2012

URBAN PIONEERS (2007) Temporary use ans urban development in Berlin, Berlin, Jovis Verlag, $192 \mathrm{p}$.

WAGNER B., Soziokultur West - Soziokultur Ost, in : Aus Politik und Zeitgeschichte, Bundeszentrale für politische Bildung, B11/2001 